\title{
Constrained Output Path-Following for Nonlinear Systems Using Predictive Control
}

\author{
Timm Faulwasser ${ }^{*, * *}$ and Rolf Findeisen* \\ * Institute for Automation Engineering, \\ Otto-von-Guericke University Magdeburg, Germany \\ ** International Max Planck Research School for Analysis, Design and \\ Optimization in Chemical and Biochemical Process Engineering \\ Magdeburg, Germany \\ E-mail: \{timm.faulwasser, rolf.findeisen\}@ovgu.de
}

\begin{abstract}
We address the problem of steering the output of a nonlinear system along a given parametrized reference path, taking input and state constraints into account. Such problems are known as output path-following problems, which typically arise in vehicle and robot control and also in process control.

We propose an efficient, continuous time, sampled-data state feedback, predictive control scheme which guarantees convergence to an output path in the presence of state and input constraints.
\end{abstract}

Keywords: Path-following, constraints, nonlinear model predictive control, semi-definite cost functions

\section{INTRODUCTION}

Classical controller design either aims at setpoint stabilization or tracking of reference signals. In general, tracking of output or state trajectories involves a timing constraint when to be where on the reference trajectory. In difference to tracking, path-following aims at driving the system towards and along a geometric curve or manifold as good as possible, which in general does not require any apriorily known timing information. Therefore the path-following controller can influence both: The system inputs as well as the reference timing when to be where on the reference path. Path-following provides a suitable framework for different applications, esspecially if following the reference precisely is more important than speed. Typical applications are: Ship or flight course control, the control of robots and CNC-machines, as well as problems arising in chemical process industries such as batch crystallization following a receipe.

Backstepping based approaches to path-following problems have been proposed inter alia in Aguiar et al. (2005, 2008); Skjetne et al. (2004). A limitation of these works is that neither input nor state constraints can be considered explicitly during the controller design. In order to overcome these limitations optimization based predictive control approaches to path-following problems have been proposed in Faulwasser and Findeisen (2009); Faulwasser et al. (2009). These works allow to consider constraints directly in a suitable, stabilizing nonlinear model predictive control (NMPC) framework but are limited to state space paths. State space paths denote reference paths which are defined in terms of the complete state variable $x \in \mathbb{R}^{n}$ while output path-following requires that only the system output should follow the reference.
From a predictive control point of view following state space paths can be considered as easier in terms of relying on available stability results than following paths defined in an output space. For state space path-following a stabilizing NMPC scheme can be derived by defining an adequate cost function (which penalizes mainly path deviation and inputs and is a strictly positive definite function of the state) and a subsequent reformulation in suitable coordinates, cf Faulwasser and Findeisen (2009); Faulwasser et al. (2009). In the case of output paths it is likewise desireable to penalize mainly path deviation and inputs in the cost function. However, since output maps $y=h(x)$ are in general not bijective one has to deal with semi-definite cost functions. Establishing a cost decrease or Barbalats Lemma like convergence condition for the complete state is difficult for such cost functions. To handle this issue one may rely on detectability conditions as in Grimm et al. (2005). Here, we follow a different approach: Instead of searching for sufficient stability conditions we pursue a set stabilization rather then a setpoint stabilization approach. Subsequently we extend previous results on predictive path-following to output path-following problems, cf Faulwasser and Findeisen (2009); Faulwasser et al. (2009). We propose a sampled-data, state feedback, quasi-infinite horizon NMPC scheme and derive sufficient conditions that guarantee output path convergence.

In Section 2 we briefly introduce the constrained output path-following problem and discuss conditions for exact path-following. Our main result is given in Section 3: An NMPC scheme that guarantees output path convergence in the presence of input and state constraints. In Section 4 we consider an autopilot for a ship as an application example. 


\section{Notation}

For any vector $y \in \mathbb{R}^{k},\|y\|$ denotes the 2-norm and the weighted norm is given by $\|y\|_{Q}=y^{T} Q y$, where $Q \in \mathbb{R}^{k \times k}$ is a positive semi-definite matrix. The image $\mathcal{I}$ of a compact set $\mathcal{S} \subseteq \mathbb{R}^{n}$ under a map $m: \mathbb{R}^{n} \mapsto \mathbb{R}^{k}$ is defined as $\mathcal{I}=m(\overline{\mathcal{S}}):=\{\forall s \in \mathcal{S} \quad s \mapsto i=m(s)\}$. The preimage $\mathcal{P} \subseteq \mathbb{R}^{n}$ of a set $\mathcal{I} \subseteq \mathbb{R}^{k}$ is denoted as $\mathcal{P}=m^{-1}(\mathcal{I}):=\left\{\forall p \in \mathbb{R}^{n}: m(p) \in \overline{\mathcal{I}}\right\}$. A solution to an ODE $\dot{x}=f(x, u)$ starting at $x_{0}$, driven by the input $u(t)$ is denoted as $x\left(t \mid x_{0}, u\right)$. If no ambiguity about the starting point can arise we may concisely write $x(t \mid u)$.

\section{THE OUTPUT PATH-FOLLOWING PROBLEM}

Throughout this work we consider nonlinear systems

$$
\begin{aligned}
& \dot{x}=f(x, u), \quad x\left(t_{0}\right)=x_{0}, \\
& y=h(x),
\end{aligned}
$$

where are $f: \mathbb{R}^{n} \times \mathbb{R}^{m} \mapsto \mathbb{R}^{n}$ and $h: \mathbb{R}^{n} \mapsto \mathbb{R}^{k}$. Furthermore, the system is subject to constraints on states $x \in \mathcal{X} \subset \mathbb{R}^{n}$ and inputs $u \in \mathcal{U} \subset \mathbb{R}^{m}$, where both constraint sets are compact and contain the origin. The initial condition belongs to a set consistent with the state constraints $x_{0} \in \mathcal{X}_{0} \subseteq \mathcal{X}$. We assume, that the vector fields $f$ and $h$ from (1) are sufficiently often continuously differentiable. Besides this $f$ has an unique continuous solution for all inital conditions in the region of interest and all inputs $u \in \mathcal{U}$.

Often one either aims at stabilizing the output $y=$ $h(x)$ around a constant setpoint against disturbances or wants to track a time-varying output reference signal. In contrast to these problems path-following aims at driving the system along a geometric reference. This reference is denoted as path $\mathcal{P}$ and given as a parametrized, geometric curve in the output space $\mathbb{R}^{k}$

$$
\mathcal{P}=\left\{\theta \in[\hat{\theta}, 0] \subset \mathbb{R} \mapsto p(\theta) \in \mathbb{R}^{k}\right\} .
$$

The scalar variable $\theta$ is the path parameter. The path is the image of $[\hat{\theta}, 0]$ under the locally bijective map $p: \mathbb{R} \mapsto \mathbb{R}^{k}$. For sake of convenience, we assume that the initial path point $\theta\left(t_{0}\right)=\theta_{0} \in[\hat{\theta}, 0)$ is known. The map $p$ is sufficiently often continuously differentiable. Hence, the path $\mathcal{P}$ is a part of a differentiable $1 \mathrm{~d}$ manifold in $\mathbb{R}^{k}$. In order to consider state constraints the following consistency is required

$$
h(\mathcal{X})=\{\forall x \in \mathcal{X} \mapsto y=h(x)\} \supset \mathcal{P} .
$$

This consistency basically ensures that for each point on the path $\mathcal{P}$ at least one $x \in \mathcal{X}$ exists s.t. $h(x) \in \mathcal{P}$. In general, the path parameter $\theta(t)$ is time dependent but its time evolution is not known apriori. The conceptual idea of path-following problems is to obtain the system inputs $u(t)$ as well as the reference timing $\theta(t)$ in the controller, e.g. Aguiar et al. (2005, 2008); Skjetne et al. (2004); Faulwasser and Findeisen (2009). Usually, the path parameter is regarded as a virtual state, whose evolution is determined through a timing law $\dot{\theta}=g(\theta, v)$. Please note, the time evolution of $\theta$ can be influenced by the virtual input $v \in \mathcal{V} \subset \mathbb{R}$. In this note we consider first order ODEs as timing laws but the consideration of higher order timing law ODEs is also possible. We restrict the choices for the timing law $g$ s.t. equivalent properties as assumed for $f$ from (1a) hold. Additionally, we suppose that the set of admissible path parameter inputs $\mathcal{V} \subset \mathbb{R}$ is compact and contains the origin. The timing law is an additional degree of freedom during the controller design.

Constrained output path-following refers to the task of steering the output of the system (1) as close as possible to the path manifold $\mathcal{P}$ from (2), and subsequently move along $\mathcal{P}$ in the direction of increasing values of $\theta$.

Definition 1 (Constrained Output Path-Following). Given the system (1) and the reference path $\mathcal{P}$ from (2) design a controller that achieves the following:

P1 Path Convergence: The system output $y=h(x)$ converges to the set $\mathcal{P}$ s.t:

$$
\lim _{t \rightarrow \infty}\left\|h\left(x\left(t \mid x_{0}, u\right)\right)-p\left(\theta\left(t \mid \theta_{0}, v\right)\right)\right\|=0 .
$$

P2 Forward Motion: The system moves along $\mathcal{P}$ in the direction of increasing values of $\theta$ s.t. for all $\theta \in[\hat{\theta}, 0): \quad \dot{\theta}(t)>0$.

P3 Constraint Satisfaction: The constraints on states $x \in \mathcal{X}$ and inputs $u \in \mathcal{U}$ are satisfied for all times.

It is reasonable to ask for sufficient conditions ensuring that a given path $\mathcal{P}$ is exactly followable by a known system (1). To this end we define path followability rigorously as follows.

Definition 2 (Exact Path Followability).

A path $\mathcal{P}$ is called exactly followable by system (1), if the system admits at least one continuous solution trajectory $x(t)$, s.t. the error variable $e(t)=h(x(t))-p(\theta(t))$ is zero for all $t \geq 0$ while the path parameter $\theta(t)$ evolves continuously with time $t$ and $\dot{\theta}>0$ holds for all $\theta \in[\hat{\theta}, 0)$.

The consistency requirement from (3) is a intuitive but merely necessary condition for exact path followability. The following theorem answers the question for sufficient conditions if the path evolution is described via a timing law ODE $\dot{\theta}=g(\theta, v)$ and the system (1) is subject to input and/or state constraints.

\section{Theorem 1}

Given the system (1) subject to input and state constraints $u \in \mathcal{U}, x \in \mathcal{X}$ and the path $\mathcal{P}$ defined by (2).

Suppose, the path $\mathcal{P}$ is consistent with the state constraints $h(\mathcal{X}) \subseteq \mathcal{P}$, and the timing law $\dot{\theta}=g(\theta, v)$ is chosen s.t. for all $\theta \in[\hat{\theta}, 0)$ and all admissible inputs $v \in \mathcal{V}: \dot{\theta}>0$.

Then $\mathcal{P}$ is exactly followable starting from $\theta_{0}=\theta\left(t_{0}\right)$, if

(i) the system state $x\left(t_{0}\right)=x_{0} \in \mathcal{X}$ is s.t. $h\left(x_{0}\right)=p\left(\theta_{0}\right)$

(ii) and admissible input signals $u \in \mathcal{U}, v \in \mathcal{V}$ exist s.t. for all $t \geq t_{0}$ the trajectories $x\left(t \mid x_{0}, u\right) \in \mathcal{X}$ and $\theta\left(t \mid \theta_{0}, v\right) \in[\hat{\theta}, 0]$ satisfy

$$
\left.\frac{\partial h}{\partial x} \cdot f(x, u)\right|_{x\left(t \mid x_{0}, u\right)}=\left.\frac{\partial p}{\partial \theta} \cdot g(\theta, v)\right|_{\theta\left(t \mid \theta_{0}, v\right)} .
$$

Proof. The proof is based on the observation that, if the systems starts at a point on the path the error variable $e(t)=h(x(t))-p(\theta(t))$ has to be stabilized at the origin while $\dot{\theta}>0$ holds. Note, by assumption the system ODE (1) as well as the timing law $g$ are such that for all admissible inputs $u \in \mathcal{U}$ and $v \in \mathcal{V}$ exist unique and continuous solutions $x(t)$ and $\theta(t)$. 
From part (i) of the theorem we know that at time $t_{0}$ we start at the point on $\mathcal{P}$ given by $\theta_{0}$. Hence $e\left(t_{0}\right)=h\left(x_{0}\right)-$ $p\left(\theta_{0}\right)=0$. The time derivative of $e(t)$ is given by

$$
\dot{e}(t)=\frac{\partial h}{\partial x} \cdot \dot{x}-\frac{\partial p}{\partial \theta} \cdot \dot{\theta} .
$$

For all $t \geq t_{0}$ condition (ii) guarantees $\dot{e}(t)=0$. Since $e\left(t_{0}\right)=0$ it follows that $e(t)=0$ for all $t \geq t_{0}$.

At the first glance these conditions seem to be rather hard to check. However, besides the real system input $u$ the virtual path parameter input $v$ and the timing law $\dot{\theta}=g(\theta, v)$ appear as additional degrees of freedom on the right hand side of (4).

\section{PROPOSED CONTROL SCHEME}

We propose to solve the output path-following problem by means of NMPC. To avoid the difficulties of real output-feedback NMPC, we assume that sampled-data state information is available to the controller. In order to distinguish between the predicted controller variables and the real system variables we denote the predicted inputs, states and outputs with $\bar{x}, \bar{u}$ etc.

At each sampling instant $t_{k}=k \delta, \delta>0, k \in \mathbb{N}$ the following optimal control problem is solved in the controller

$$
\underset{\bar{u}(\cdot), \bar{v}(\cdot)}{\operatorname{minimize}} J\left(t_{k}, \bar{e}, \bar{\theta}\right)
$$

the cost functional is defined as

$$
J(\cdot)=\int_{t_{k}}^{t_{k}+T} F(\bar{e}, \bar{\theta}, \bar{u}, \bar{v}) d \tau+\left.E(\bar{e}, \bar{\theta})\right|_{t_{k}+T},
$$

where $F(\cdot): \mathbb{R}^{k} \times \mathbb{R} \times \mathcal{U} \times \mathcal{V} \mapsto \mathbb{R}_{\geq 0}$. The term $E(\cdot): \mathbb{R}^{k} \times$ $\mathbb{R} \mapsto \mathbb{R}_{>0}$ is referred to as terminal cost. The positive constant $T$ is called prediction horizon. This optimal control problem is subject to the system model and further constraints

$$
\begin{aligned}
& \dot{\bar{x}}=f(\bar{x}, \bar{u}), \quad \bar{x}\left(t_{k}\right)=x\left(t_{k}\right), \\
& \dot{\bar{\theta}}=g(\bar{\theta}, \bar{v}), \quad \bar{\theta}\left(t_{k}\right)=\bar{\theta}\left(t_{k} \mid \bar{\theta}\left(t_{k-1}\right)\right), \\
& \bar{e}=h(\bar{x})-p(\bar{\theta}), \\
& \forall \tau \in\left[t_{k}, t_{k}+T\right]: \quad \bar{x}(\tau) \in \mathcal{X}, \bar{u}(\tau) \in \mathcal{U}, \\
& \forall \bar{\theta}(\tau) \in[\hat{\theta}, 0): \quad \dot{\bar{\theta}}(\tau)>0, \bar{v}(\tau) \in \mathcal{V}, \\
& \bar{x}\left(t_{k}+T\right) \in \mathcal{E} .
\end{aligned}
$$

Equation (5h) requires that at the end of each prediction the predicted state $\bar{x}\left(t_{k}+T\right)$ has to be inside a terminal region $\mathcal{E}$. Equation $(5 \mathrm{~d})$ states that the path parameter $\theta$ is regarded as a virtual state of the NMPC scheme. Its dynamics are described through the timing law $g$ and can be influenced by the virtual input $v$, where $v$ is a decision variable of the minimization. Please note, while at each sampling instance the measured state information $x\left(t_{k}\right)$ serves as initial condition for $(5 c)$ the initial conditions for the timing law (5d) are taken from the evaluation of the last predicted trajectory $\bar{\theta}\left(t \mid \bar{\theta}\left(t_{k-1}\right)\right)$ at $t_{k}$. Equation (5e) specifies the path-following error. We point out that not the complete state but only the predicted error $\bar{e}(t)$ is penalized in the cost function $F$ and the terminal cost $E$. The optimal solution of problem (5) is denoted as $J^{\star}(\cdot)$ and leads to optimal input trajectories $\bar{u}^{\star}\left(t \mid x\left(t_{k}\right)\right)$ and $\bar{v}^{\star}\left(t \mid x\left(t_{k}\right)\right)$. Finally $\bar{u}^{\star}(\cdot)$ is applied to the system such that for all $t \in\left(t_{k}, t_{k}+\delta\right]: u(t)=\bar{u}^{\star}\left(t \mid x\left(t_{k}\right)\right)$.
In two crucial aspects the proposed scheme differs from standard NMPC approaches. First, the path-following specific constraints capture an additional degree of freedom of the controller design, since the timing law (5d) is not given apriori but rather can be chosen. Second, the cost $F(\bar{e}, \bar{\theta}, \bar{u}, \bar{v})$ is in general a positive semi-definite function of the predicted system state $\bar{x}$ since $\bar{e}=h(\bar{x})-p(\bar{\theta})$. Actually, the second aspect turns out to be a major issue. As previously investigated inter alia by Grimm et al. (2005) it is possible to guarantee stability of predictive control schemes subject to semi-definite cost functions if certain detectability assumptions hold for $F(\cdot)$. But the constrained output path-following defined above does not require to guarantee stability of the state. Rather we need to ensure that the output $y=h(x)$ converges to $\mathcal{P}$ and follows it along while the constraints $u \in \mathcal{U}$ and $\dot{\theta}>0$ are respected. Furthermore, we want to ensure that the state remains bounded in $x \in \mathcal{X}$.

In order to derive an output convergence result for the proposed control scheme we require that the cost function $F(e, \cdot)$ is lower bounded by a class $\mathcal{K}$ function $\alpha(e)$ and that $F(0,0,0,0)=0$.

\section{Definition 3 (Path Consistent State Set).}

Given the system (1) subject to input and state constraints and the path $\mathcal{P}$ from (2). Consider a subset $\Gamma$ of the preimage $h^{-1}(\mathcal{P}) \subset \mathbb{R}^{n}$, where $h: \mathcal{X} \mapsto \mathbb{R}^{k}$ is the output map $y=h(x)$ from (1b). For all $x \in \Gamma$ the following conditions have to hold:

(i) For all $\theta \in[\hat{\theta}, 0]$ exist $x \in \Gamma$, s.t. $h(x)=p(\theta)$.

(ii) There exist admissible inputs $u_{\Gamma} \in \mathcal{U}$ and $v_{\Gamma} \in \mathcal{V}$ s.t. condition (ii) of Theorem 1 holds: The system follows $\mathcal{P}$ exactly along in forward direction.

Basically, the path consistent state set $\Gamma$ is the set of all states $x$, where the output $y=h(x)$ is on the path and the admissible inputs can move the output exactly along the path. Although the output map $h$ from (1b) is continuous, there is in general no guarantee that either the preimage of the path $h^{-1}(\mathcal{P})$ is compact nor that it can be rendered positively invariant by admissible inputs. Hence, we make the following standing assumption.

\section{Assumption 1}

The set of consistent states $\Gamma$ is nonempty, simply connected and compact.

The existence of a nonempty, simply connected and compact set $\Gamma$ ensures that path can be followed exactly by admissible inputs, since for all $x \in \Gamma$ Theorem 1 has to hold. While the existence of $\Gamma$ ensures path followability, its connected- and compactness are assumed for technical reasons. Figure 1 illustrates the main idea behind the set $\Gamma$ from Definition 3 and Assumption 1. The right hand side depicts the consistency requirement between the sets $h(\mathcal{X})$ and $\mathcal{P}$ as previously stated in $(3)$. The left hand side illustrates the relations between the preimage $h^{-1}(\mathcal{P})$, the state constraint set $\mathcal{X}$ and $\Gamma$. Assumption 1 states that the conjunction $X \cap h^{-1}(\mathcal{P})$ is nonempty and that a compact set $\Gamma \subseteq\left\{X \cap h^{-1}(\mathcal{P})\right\}$ exists. Furthermore, Assumption 1 can be used to deduce the existence of a suitable end penalty $E(\cdot)$ which will be later used to bound the cost-togo of the proposed control scheme and hence to guarantee path convergence. 


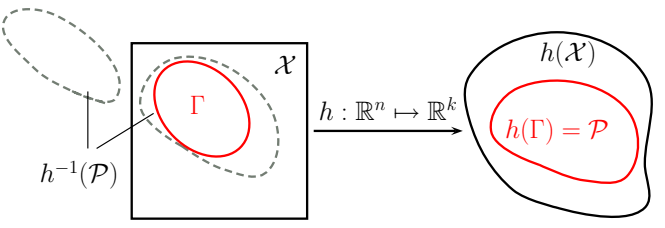

Fig. 1. Relations between the constraint set $\mathcal{X}$, the path consistent set $\Gamma$ and the preimage $h^{-1}(\mathcal{P})$.

\section{Lemma 1}

Given the system (1), a path $\mathcal{P}(2)$, the set $\Gamma$ from Definition 3 and provided that Assumption 1 holds.

\section{Suppose}

(i) that the admissible input signals $u_{\Gamma} \in \mathcal{U}$ and $v_{\Gamma} \in \mathcal{V}$ exist,

(ii) and for an $\epsilon>0$ and all $\theta \in[\epsilon, 0]$ it holds that $g\left(\theta, v_{\Gamma}\right) \geq F\left(0, \theta, u_{\Gamma}, v_{\Gamma}\right) \geq 0$.

Then there exists a continuously differentiable, positive semi-definite function $E(\theta):[\hat{\theta}, 0] \mapsto \mathbb{R}_{\geq 0}$ where $E(0)=0$ s.t. for all $x \in \Gamma$

$$
\frac{\partial E(\theta)}{\partial \theta} \cdot \dot{\theta}+F\left(0, \theta, u_{\Gamma}, v_{\Gamma}\right) \leq 0 .
$$

Proof. The proof is built on the idea that the pathparameter $\theta$ can be considered as a suitable alternative time variable. Due to Assumption 1 the set $\Gamma$ is compact and nonempty. From Assumption 1 we know that the input signals $u_{\Gamma}(t), v_{\Gamma}(t)$ render $\Gamma$ positively invariant and $e=0$ holds for all $x \in \Gamma$.

Due to the properties required for $g$ the input $v_{\Gamma}$ ensures that the map $t \mapsto \theta(t)$ is unique and continuous. Since $\dot{\theta}>0$ holds for all $\theta \in[\hat{\theta}, 0)$ the map $t \mapsto \theta(t)$ is uniquely invertible to $\theta \mapsto t(\theta)$ for all $\theta \in[\hat{\theta}, 0)$. Due to its continuity it is also uniquely invertible on $[\hat{\theta}, 0]$. Hence the time parametrization of the inputs along the path $\mathcal{P}$ may be expressed in terms of the path parameter $\theta$ and is written as $t(\theta)$.

Since the constraint sets $\mathcal{U}, \mathcal{V}$ and $[\hat{\theta}, 0]$ are compact and $F(\cdot)$ is continuous in all arguments it follows that

$$
\forall t \geq 0, \forall x \in \Gamma: \quad F\left(0, \theta(t), u_{\Gamma}(t), v_{\Gamma}(t)\right)<\infty .
$$

Relying on the time parametrization $t(\theta)$ this can be rewritten as

$$
\forall \theta \in[\hat{\theta}, 0], \forall x \in \Gamma: \quad F\left(0, \theta, u_{\Gamma}(\theta), v_{\Gamma}(\theta)\right)<\infty .
$$

Consider the following integral along the path

$$
0 \leq \int_{\hat{\theta}}^{0} \frac{F\left(0, \eta, u_{\Gamma}(\eta), v_{\Gamma}(\eta)\right)}{\dot{\theta}(\eta)} d \eta,
$$

and split it into the parts

$$
0 \leq \int_{\hat{\theta}}^{\epsilon} \frac{F(\cdot)}{\dot{\theta}(\eta)} d \eta+\int_{\epsilon}^{0} \frac{F(\cdot)}{\dot{\theta}(\eta)} d \eta .
$$

The existence and boundedness of the first integral can be established without any difficulty, since $\dot{\theta}>0$ and $F(\cdot)$ is bounded. Due to part (ii) of the lemma $F(\cdot)$ converges faster to zero than $\dot{\theta}=g\left(\theta, v_{\Gamma}\right)$ and hence the second integral exists and is bounded. It follows that for all $\theta \in[\hat{\theta}, 0]$

$$
E(\theta)=-\beta \int_{\theta}^{0} \frac{F\left(0, \eta, u_{\Gamma}(\eta), v_{\Gamma}(\eta)\right)}{\dot{\theta}(\eta)} d \eta, \quad \beta \in[1, \infty)
$$

is a suitable terminal cost $E(\theta)$ which fulfills condition (6) for all $x \in \Gamma$.

The lemma offers some insights into suitable terminal penalites and terminal regions. $\Gamma$ is a reasonable choice for the terminal region $\mathcal{E}$ from $(5 \mathrm{~h})$. Mainly, Lemma 1 provides a suitable way to calculate an end penalty $E(\cdot)$ if $\mathcal{E}=\Gamma$. Please note, the conditions required are not as strict as they may seem, since the timing law $g(\cdot)$ is chosen during the controller design. However, $\Gamma$ is a minimal or conservative choice for $\mathcal{E}$, since the pair end penalty $\mathcal{E}$ and terminal region $\Gamma$ can be regarded as a zero terminal path constraint, which was initially developed for the case of state space paths in Faulwasser et al. (2009).

Since we want to consider cases, where the system does not start on the path $\mathcal{P}$ (or more precisely inside $\Gamma$ ), it is also necessary to assume that the path can be reached in finite time by admissible inputs.

\section{Assumption 2}

For all initial conditions $x_{0} \in \mathcal{X}_{0}$ exist admissible input signals $u_{0} \in \mathcal{U}, v_{0} \in \mathcal{V}$ and a positive time $\tilde{T}<\infty$, s.t. the solution $x\left(t \mid x_{0}, u_{0}\right)$ is contained in $\Gamma \subset \mathcal{X}$ for all $t \geq \tilde{T}$.

With respect to Assumption 2 the system can be driven by means of admissible inputs in finite time from any point in $\mathcal{X}_{0}$ to $\Gamma$. Obviously, the prediction horizon of the optimal control problem (5) has to be chosen s.t. $T \geq \tilde{T}$. Assumptions 1 and 2 are not as restrictive it seems. Basically, they ensure that the considered output path-following problem is reasonably structured: The path is exactly followable under the given input and state constraints (Ass. 1) and the optimal control problem (5) is initially feasible for all $x_{0} \in \mathcal{X}_{0}$ (Ass. 2). The existence of an (in general non-unique) optimal solution to the optimal control problem (5) can be ensured, if convexity assumptions are made for the velocity sets of the system dynamics (1a) and the timing law (5d), cf Fontes (2001). For sake of a concise presentation we assume that the optimal control problem has an optimal solution which is actually attained.

\section{Main Result}

Under the given assumptions we are now ready to state the main result of this note providing convergence conditions for the output path-following problem.

Theorem 2 (Output Path Convergence).

Given the constrained output path-following problem from Definition 1 for a system of type (1), a path of form (2) and provided Assumptions $1 \& 2$ hold.

Suppose, that conditions (i) and (ii) of Lemma 1 are satisfied. Then there exists a compact (terminal) region $\mathcal{E} \supseteq \Gamma$ and a semi-definite terminal cost $E(\bar{e}, \bar{\theta}) \in \mathcal{C}^{1}$ s.t. the application of the predictive path-following scheme defined by (5) guarantees that the constrained output path-following problem (P1-P3) are satisfied. All system 
states $x$ remain bounded in $\mathcal{X}$ and the system output converges to the path $\mathcal{P}$ s.t.

$$
\lim _{t \rightarrow \infty}\left\|h\left(x\left(t \mid x_{0}, u(\cdot)\right)\right)-p\left(\theta\left(t \mid \theta_{0}, v(\cdot)\right)\right)\right\|=0 .
$$

Proof. The proof follows along the ideas presented e.g. in Findeisen (2006); Fontes (2001) and we sketch here only the three main steps: First, we discuss the feasibility of the optimal control problem (5). Second, we know from Lemma 1 that Assumption 1 suffices to guarantee that the set $\Gamma$ can always be used as terminal region s.t. the cost to go can be upper bounded by a terminal cost $E(\theta)$. And finally, we line out how one can apply Barbalats Lemma to proof convergence.

From Assumption 1 we know that the problem (5) admits a feasibile solution $u_{\Gamma}, v_{\Gamma}$ for all $x \in \Gamma$. And from Assumption 2 we know that from all $x_{0} \in \mathcal{X}_{0}$ the set $\Gamma$ can be reached by admissible inputs $u_{0}, v_{0}$ in some finite time $T>0$. Since the nominal case is considered the concatenation

$$
(u, v)= \begin{cases}\left(u_{0}, v_{0}\right) & \text { for } t \leq T \\ \left(u_{\Gamma}, v_{\Gamma}\right) & \text { for } t>T\end{cases}
$$

yields input signals which guarantee that the constrained output path-following problem is solved. Following along the ideas presented in Fontes (2001) it is straight forward to conclude that Assumptions 1 \& 2 guarantee the feasibility of the optimal control problem (5) at every sampling instant $t_{k}$.

We point out, that without loss of generality the choice of timing laws can be restricted to the set of functions $g$ s.t. for all $\theta \in[\hat{\theta}, 0)$ and all $v \in \mathcal{V}$ it holds $g(\theta, v)>0$. Hence we can assume that the forward movement condition P2 of the output path-following problem is satisfied.

The following lemma establishes that the optimal value function is decreasing from sampling instance to sampling instance. Its proof follows directly the ideas presented in Fontes (2001).

\section{Lemma 2}

Given Assumptions $1 \& 2$ and suppose that the set $\Gamma$ and the terminal penalty from Lemma 1 are used in the optimal control problem (5). Then for $k \in \mathbb{N}$ it holds

$$
J^{\star}\left(t_{k+1}, e^{\star}, \theta^{\star}\right)-J^{\star}\left(t_{k}, e^{\star}, \theta^{\star}\right) \leq-\int_{t_{k}}^{t_{k+1}} \alpha\left(e^{\star}(s)\right) d s .
$$

From Assumption 2 it can be deduced that the value function $J^{\star}\left(t_{0}, \cdot\right)$ is upper bounded by a finite scalar. It follows that for all $t>t_{0}$

$J^{\star}\left(t_{k+1}, e^{\star}, \theta^{\star}\right)-\int_{t_{k}}^{t_{k+1}} \alpha\left(e^{\star}(s)\right) d s \leq J^{\star}\left(t_{0}, e^{\star}\left(t_{0}\right), \theta^{\star}\left(t_{0}\right)\right)$.

Since the properties of the dynamics $f, k$ and the maps $h, p$ are s.t. the uniform continuity of $e(t)=h(x(t))-p(\theta(t))$ is ensured. Using Lemma 2 it follows by Barbalats Lemma that $\lim _{t \rightarrow \infty} e(t)=0$.

\section{Remarks}

It should be clearly noted, that the proposed control scheme aims on convergence of the output $y=h(x)$ to the path and not on Lyapunov state stability. This means that Theorem 2 in principle allows cases where the output converges to the path while the states might move through $\mathcal{X}$. This mainly stems from the fact that intuitive cost functions for the output path-following problem penalize the deviation from the reference path and inputs but not necessarily the complete state.

Furthermore, the results given above share a difficulty with various other quasi-infinite horizon NMPC schemes: The computation of suitable terminal regions and end penalties is rather hard. Referring to the proposed control scheme this boils down to the question of pinpointing the path consistent state set $\Gamma$, since Lemma 1 tell us how to compute a suitable terminal penalty if $\Gamma$ is known

A straightforward but conservative choice is the off-line computation of a path consistent trajectory $x\left(t \mid x_{0}, u\right)$ such that $h\left(x\left(t \mid x_{0}, u\right)\right)=p\left(\theta \mid \theta_{0}, v\right)$. The terminal constraint (5h) is then replaced by $\bar{x}\left(t_{k}+T \mid x\left(t_{k}\right)\right)=x\left(t_{k}+T \mid x_{0}, u\right)$. This choice will lead to a path convergent NMPC scheme but the controller will always try to pinpoint the path evolution to the trajectory determined off-line.

If the considered system (1) is differentially flat, s.t. the output (1b) is a flat output (see Fliess et al. (1995) for details), then there exists a map $\phi\left(y, \dot{y}, \ldots, y^{(l)}\right)=x$ which allows to reconstruct the state from the output and a finite number of its time derivatives. In this case and if the path $\mathcal{P}$ is constructed by a map $p(\theta) \in C^{m \geq l+1}$ one should use an $l+1$-th order ODE as timing law and determine $\Gamma$ by utilizing the map $\phi$. However, the system property of differential flatness is only sufficient but not necessary for the existence of such a map $\phi$.

\section{EXAMPLE: SHIP AUTOPILOT}

We consider an output path-following problem as it appears in ship control as an example. The dynamics of a ship are given by

$$
\left(\begin{array}{c}
\dot{x}_{1} \\
\dot{x}_{2} \\
\dot{x}_{3} \\
\dot{x}_{4}
\end{array}\right)=\left(\begin{array}{c}
w \cos x_{3}-L w x_{4} \sin x_{3} \\
w \sin x_{3}+L w x_{4} \cos x_{3} \\
x_{4} \\
\frac{1}{\tau}\left(-x_{4}+K u\right)
\end{array}\right) .
$$

The states $x_{1}$ and $x_{2}$ describe the position of the ship with respect to a global coordinate system. $x_{3}$ is the orientation of the ship with respect to the $x_{1}$ direction. $x_{4} \in[-0.2,0.2]$ is the rotation rate of the ship. The input $u \in[-0.6,0.6]$ is the rudder angle, $\tau=22.32, K=-0.036, w=3$ and $L=3.93$ are constants, and $w$ is the forward velocity of the ship. The considered output is given as $y=\left(x_{1}, x_{2}\right)^{T}$, where the components describe to the position in the $x_{1}-$ $x_{2}$ plane. The path $\mathcal{P}$ to be followed is $p(\theta)=(\theta, \rho(\theta))^{T}$, where $\rho(\theta)=a_{1} \sin a_{2} \theta$ and $a_{1}=-10^{3}, a_{2}=1.5 \cdot 10^{-3}$. The path parameter $\theta$ starts at $\theta_{0}=-4000$. Its evolution is $\dot{\theta}=-10^{-4} \theta+v$, where $v \in[0,4]$. This choice guarantees that $\dot{\theta}>0$ holds for all $v \in \mathcal{V}$.

The path-following error to be stabilized is $e(t)=y(t)-$ $p(\theta(t))$. We consider the cost function $F(e)=\|e\|_{Q}, \quad Q=$ $10^{5} \operatorname{diag}(1,1)$. The prediction horizon is set to $T=500 \mathrm{~s}$ and the control horizon is $\delta=60 \mathrm{~s}$. Since neither $\theta$ nor the inputs are present in the considered cost, it follows from Lemma 1 that the terminal penalty can be neglected without loss of path convergence, if the path consistent state set $\Gamma$ is known. Actually, one can show that the 
(a) $y_{1}-y_{2}$ plane

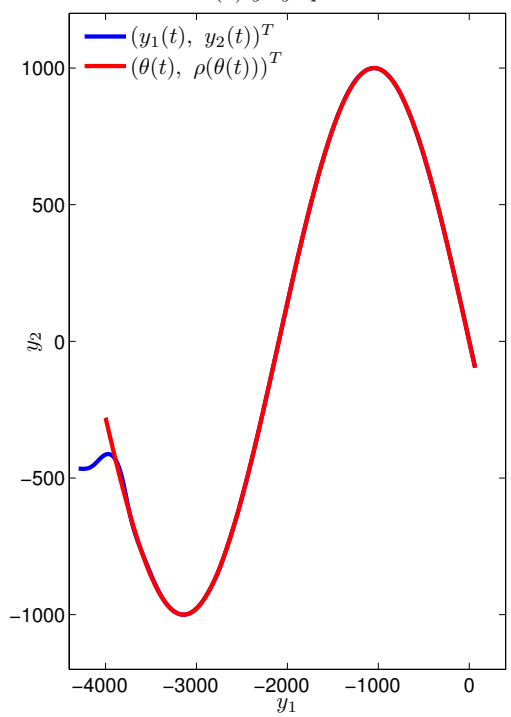

(b) States $x_{1}(t), x_{2}(t)$

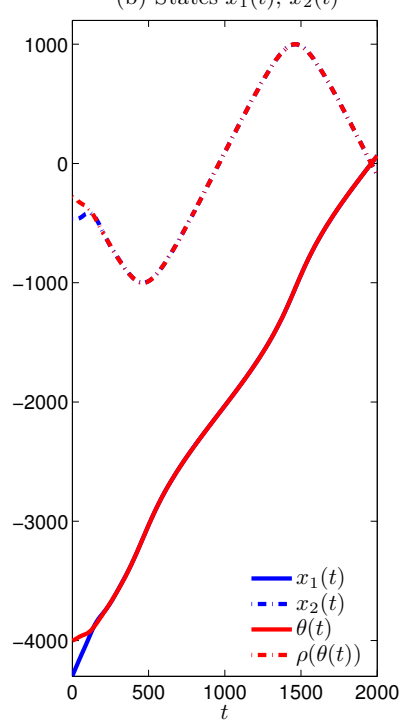

(c) States $x_{3}(t), x_{4}(t)$

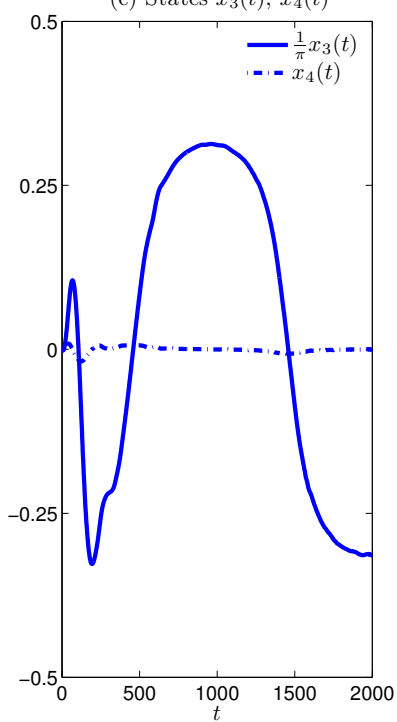

(d) Inputs

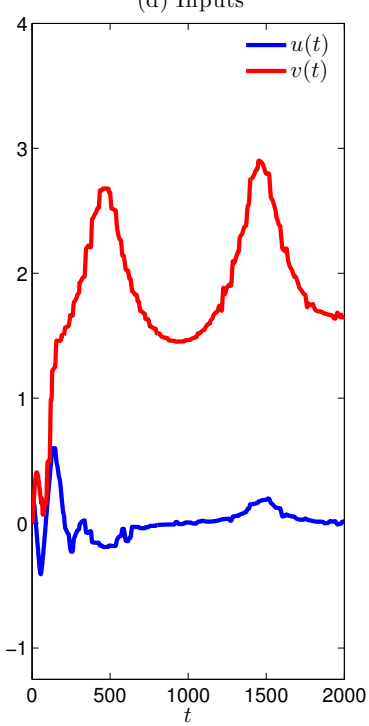

Fig. 2. Simulation results: (a) ship movement in $y_{1}-y_{2}$-plane, state evolutions in (b) \& (c) and input signals in (d).

considered path $\mathcal{P}$ is exactly followable by the constrained system (8). To avoid the computation of the set $\Gamma$ we utilize the exact path-following condition of Theorem 1 and approximate $\Gamma$ by the terminal constraints $\| \dot{e}\left(t_{k}+\right.$ $T) \| \leq \epsilon, \epsilon=0.2$. These conditions ensure, that at the end of each prediction horizon the system is sufficiently close to the path, while the output velocity vector is nearly the same as the current reference velocity vector. The computations were carried out using PROPT-TOMLAB under MATLAB 7, see Rutquist and Edvall (2009) for details. The simulation results for the initial condition $x_{0}=(-4296,-466,-0.75,0)^{T}$ are shown in Figure 2 . The ship movement converges rapidly to the path and follows it along. As depicted in parts (c) and (d) of the figure the constraints on the system input $u \in[-0.6,0.6]$ and $x_{4} \in[-0.2,0.2]$ are satisfied.

\section{CONCLUSIONS AND OUTLOOK}

In this note we propose a predictive control approach to output path-following problems in the presence of input and state constraints. The given results state sufficient conditions that guarantee the convergence of the system output to an apriorily known reference path. Further works should focus on two main aspects: On the one hand the calculation of suitable terminal regions and the path consistent set of states needs to be investigated more closely. On the other hand robustness issues have not been considered for predictive path-following, yet.

\section{ACKNOWLEDGEMENTS}

The authors gratefully acknowledge the fruitful discussions with Friedrich von Haeseler, Paolo Varutti and Benjamin Kern.

\section{REFERENCES}

Aguiar, A., Hespanha, J., and Kokotovic, P. (2005). Pathfollowing for nonminimum phase systems removes per- formance limitations. IEEE Trans. Automat. Contr., 50(2), 234-239.

Aguiar, A., Hespanha, J., and Kokotovic, P. (2008). Performance limitations in reference tracking and path following for nonlinear systems. Automatica, 44(3), 598610.

Faulwasser, T. and Findeisen, R. (2009). Nonlinear model predictive path-following control. In L. Magni, D. Raimundo, and F. Allgöwer (eds.), Nonlinear Model Predictive Control: Towards New Challenging Applications, Lecture Notes in Control and Information Sciences 384, 335-343. Springer, Berlin, Heidelberg.

Faulwasser, T., Kern, B., and Findeisen, R. (2009). Model predictive path-following for constrained nonlinear systems. In Proc. 48th IEEE Conference on Decision and Control held jointly with the 2009 28th Chinese Control Conference CDC/CCC 2009, 8642-8647.

Findeisen, R. (2006). Nonlinear Model Predictive Control: A Sampled-Data Feedback Perspective. Fortschr.-Ber. VDI Reihe 8 Nr. 1087, VDI Verlag, Düsseldorf.

Fliess, M., Lévine, J., Martin, P., and Rouchon, P. (1995). Flatness and defect of non-linear systems: introductory theory and examples. Int. J. Contr., 61(6), 1327-1361.

Fontes, F. (2001). A general framework to design stabilizing nonlinear model predictive controllers. Sys. Contr. Lett., 42(2), 127-143.

Grimm, G., Messina, M.J., Tuna, S.E., and Teel, A.R. (2005). Model predictive control: for want of a local control lyapunov function, all is not lost. IEEE Trans. Automat. Contr., 50(5), 546-558.

Rutquist, P. and Edvall, M. (2009). PROPT-Matlab optimal control software. Tomlab Optimization Inc.

Skjetne, R., Fossen, T., and Kokotovic, P. (2004). Robust output maneuvering for a class of nonlinear systems. Automatica, 40(3), 373-383. 